\title{
BMJ Open Proinsulin in the identification and risk stratification of gestational diabetes mellitus: study protocol for a prospective, longitudinal cohort study
}

\author{
Rajesh Peter, Dominic Bright, Wai-Yee Cheung, Stephen D Luzio, \\ Gareth J Dunseath
}

To cite: Peter R, Bright $D$, Cheung W-Y, et al. Proinsulin in the identification and risk stratification of gestational diabetes mellitus: study protocol for a prospective, Iongitudinal cohort study. BMJ Open 2018;8:e022571. doi:10.1136/ bmjopen-2018-022571

- Prepublication history for this paper is available online. To view these files, please visit the journal online (http://dx.doi org/10.1136/bmjopen-2018022571).

Received 23 February 2018 Revised 31 May 2018 Accepted 10 July 2018

D) Check for updates

(c) Author(s) (or their employer(s)) 2018. Re-use permitted under CC BY-NC. No commercial re-use. See rights and permissions. Published by BMJ.

Diabetes Research Group, Swansea University, Swansea, UK

Correspondence to Professor Stephen D Luzio; S.Luzio@swansea.ac.uk

\section{ABSTRACT}

Introduction Gestational diabetes mellitus (GDM) is a common metabolic disorder occurring in up to $10 \%$ of pregnancies in the western world. Most women with GDM are asymptomatic; therefore, it is important to screen, diagnose and manage the condition as it is associated with an increased risk of maternal and perinatal complications. Diagnosis of GDM is made in the late second trimester or early third trimester because accurate diagnosis or risk stratification in the first trimester is still lacking. An increase in serum proinsulin may be seen earlier in pregnancy and before a change in glycaemic control can be identified. This study will aim to establish if fasting proinsulin concentrations at 16-18 weeks gestation will help to identify or risk stratify high-risk pregnant women with GDM.

Methods and analysis This is a prospective, longitudinal cohort study. Two oral glucose tolerance tests will be carried out at 16-18 and 24-28 weeks gestation in 200 pregnant women with at least one risk factor for GDM (body mass index $>30 \mathrm{~kg} / \mathrm{m}^{2}$, previous macrosomic baby $(>4.5 \mathrm{~kg}$ ), previous gestational diabetes, first degree relative with type 2 diabetes mellitus) recruited from antenatal clinics. Blood samples will be taken fasting and at $30 \mathrm{~min}, 1$ and 2 hours following the $75 \mathrm{~g}$ glucose load. In addition, a fasting blood sample will be taken 6 -weeks post delivery. All samples will be analysed for glucose, insulin, C peptide and proinsulin. Recruitment began in November 2017. Optimal cut-off points for proinsulin to diagnose gestational diabetes according to National Institute for Health and Care Excellence (2015) criteria will be established by the receiver operating characteristic plot and sensitivity and specificity will be calculated to assess the diagnostic accuracy of proinsulin at 16-18 weeks gestation.

Ethics and dissemination This study received ethical approval from the Wales Research Ethics Committee (Panel 6) (Ref. 17/WA/0194). Data will be presented at international conferences and published in peerreviewed journals.

Trial registration number ISRCTN16416602; Pre-results

\section{INTRODUCTION}

Gestational diabetes mellitus (GDM) is a common metabolic disorder occurring in up
Strengths and limitations of this study

- This is a prospective, longitudinal cohort study recruiting at a single site.

- It is the first study to assess the use of proinsulin as a biomarker for the identification and risk stratification of gestational diabetes mellitus in early pregnancy in women with at least one risk factor for gestational diabetes mellitus.

- Proinsulin will be measured using sensitive and highly specific immunoassays, eliminating the influence of other beta-cell products observed with previous proinsulin assays.

- The recruitment of 200 pregnant women is planned. The study has been designed to be sufficiently powered to compare the early screening approach with the detection of gestational diabetes mellitus at 24-28 weeks of gestation.

- The use of proinsulin will be evaluated in a cohort of women at high risk of developing gestational diabetes mellitus and the study will be underpowered as a screening tool for all pregnant women.

to $10 \%$ of pregnancies in the Western world. ${ }^{1}$ GDM is defined as any degree of carbohydrate intolerance with onset or first recognition during pregnancy. Most women with GDM are asymptomatic; hence, it is important to screen, diagnose and manage the condition as it is associated with an increased risk of maternal and perinatal complications such as pre-eclampsia, macrosomia, shoulder dystocia and neonatal hypoglycaemia. Diagnosis of GDM is made in the late second trimester or early third trimester because accurate diagnosis or risk stratification in the first trimester is still lacking, and in the UK women with a high risk of GDM are currently offered an oral glucose tolerance test (OGTT) at 24-28 weeks gestation. ${ }^{2}$

The risk factors that predispose to the development of GDM include body mass index $(\mathrm{BMI})>30 \mathrm{~kg} / \mathrm{m}^{2}$, previous macrosomic baby 
$(>4.5 \mathrm{~kg})$, previous gestational diabetes, family history of type 2 diabetes mellitus (T2DM) (first degree relative with diabetes) and certain ethnic groups. Current diagnostic criteria are a fasting plasma glucose concentration of $\geq 5.6 \mathrm{mmol} / \mathrm{L}$ or a 2 hour value of $\geq 7.8 \mathrm{mmol} / \mathrm{L}^{2}{ }^{2}$ Glycosylated haemoglobin $\left(\mathrm{HbAl}_{\mathrm{c}}\right.$ ) cannot be used to diagnose GDM as HbA1 is insufficiently sensitive to substitute for OGTT as a screening test. ${ }^{3}$

Women who develop GDM have a high risk of future-compromised glycaemic control. A review of published studies indicated a 7.43-fold increase risk of postpartum diabetes in women with GDM compared with women with healthy glycaemic control during pregnancy ${ }^{4}$ and the incidence of postpartum diabetes in North America and Europe has indicated a prevalence rate of between $30 \%$ and $50 \%$ up to 15 years' follow-up..$^{5-7}$

Normal pregnancy is accompanied by a progressive increase in insulin resistance that begins midway through pregnancy and progresses through the second and third trimesters with resultant increase in insulin secretion to compensate for the acquired resistance. The levels of insulin resistance are not too dissimilar to that seen in individuals with T2DM. ${ }^{8}$ GDM then develops when the insulin supply is no longer adequate to maintain normal blood glucose regulation.

Proinsulin is a precursor molecule for insulin and is synthesised by the pancreatic beta cells. Proinsulin is an 86 amino acid peptide, incorporating the $\mathrm{A}$ and $\mathrm{B}$ chains of insulin in addition to $\mathrm{C}$ peptide between amino acid residues 31 and 65 . Under normal circumstances, virtually all proinsulin is cleaved at residues $32-33$ and 65-66 to produce $\mathrm{C}$ peptide and insulin, although a small amount of intact proinsulin may also be released into the circulation along with des 31-32 split proinsulin and 32-33 split proinsulin. In the presence of insulin resistance, pancreatic beta-cell function is affected with disproportionately more proinsulin (both intact and split) being secreted compared with insulin as seen in subjects with T2DM. ${ }^{9}$

Previous studies investigating serum proinsulin measurements in pregnancy have studied pregnant women irrespective of individual risk which may account for varied findings. ${ }^{10-12}$ However, studies comparing the proinsulin concentrations of healthy pregnant women and those who have gestational diabetes have found that proinsulin concentrations at fasting are significantly elevated in GDM compared with control subjects. ${ }^{13} 14$ These studies however did not assess the use of proinsulin as a biomarker for GDM; rather they compare women already diagnosed with GDM with proinsulin measured only at 24-28 weeks. Therefore it is possible that an increase in serum proinsulin may be seen earlier in pregnancy and before a change in glycaemic control can be identified. Specifically targeting pregnant women that are high risk may make serum proinsulin measurements more sensitive and specific to identify those that will develop GDM at an earlier stage in their pregnancy and subsequently suitable for earlier intervention.

\section{AIMS AND OBJECTIVES}

This study will aim to establish if fasting proinsulin concentrations at 16-18 weeks gestation will help to identify or risk stratify high-risk pregnant women with GDM diagnosed according to National Institute for Health and Care Excellence (2015) criteria. ${ }^{2}$ It will also seek to establish if $30 \mathrm{~min}$ and/or 1 and 2 hour post oral glucose load proinsulin measurements at 16-18 weeks gestation can discriminate women with gestational diabetes and predict which women will need insulin to control hyperglycaemia. The relationship of the various risk factors to plasma proinsulin levels will also be evaluated.

The primary objective of the study is to test the hypothesis that fasting intact proinsulin measurements at 16-18 weeks gestation will discriminate or risk stratify gestational diabetes (diagnosed from an OGTT at 24-28 weeks) from women with normal glucose tolerance.

The secondary objectives are:

- To test the hypothesis that $30 \mathrm{~min}, 1$ and 2 hour post $75 \mathrm{~g}$ oral glucose load, proinsulin measurements at 16-18 weeks gestation can predict those women subsequently diagnosed with gestational diabetes at 24-28 weeks.

- To test the hypothesis that fasting, $30 \mathrm{~min}$ and/or 1 and 2 hour post oral glucose load, proinsulin measurements at 16-18 weeks gestation can predict those women with gestational diabetes that will need insulin during pregnancy.

- To study the relationship of various risk factors to plasma proinsulin concentrations and gestational diabetes in the second and third trimesters of pregnancy.

\section{METHODS AND ANALYSIS \\ Study design}

This is a prospective, longitudinal cohort study (table 1). Study recruitment started on 14 November 2017 and the study is expected to last until December 2019.

Two OGTTs will be carried out at 16-18 and 24-28 weeks gestation in 200 pregnant women with at least one risk factor for GDM (BMI $>30 \mathrm{~kg} / \mathrm{m}^{2}$, previous macrosomic baby $(>4.5 \mathrm{~kg})$, previous gestational diabetes, first degree relative with T2DM) recruited from antenatal clinics within Abertawe Bro Morgannwg University Health Board, Wales. Women of ethnic origin considered to be high risk will need to have another mentioned risk factor to be eligible for inclusion in the study. Blood samples will be taken fasting and at $30 \mathrm{~min}, 1$ and 2 hours following the $75 \mathrm{~g}$ glucose load. In addition, a fasting blood sample will be taken at 6-weeks post delivery. All samples will be analysed for glucose, insulin, $\mathrm{C}$ peptide and proinsulin. Both intact proinsulin and total proinsulin (the sum of 
Table 1

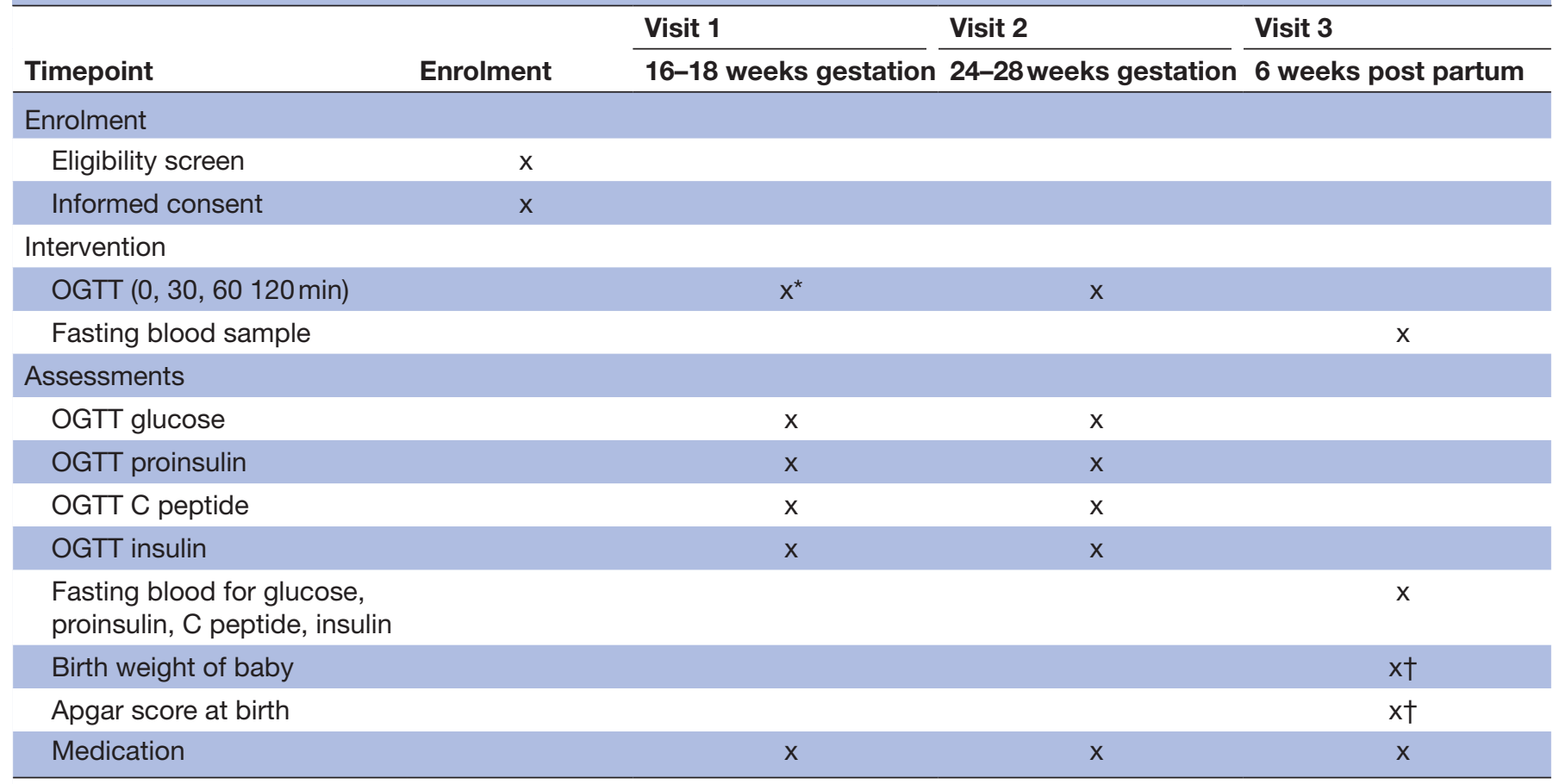

*Participants exceeding the National Institute for Health and Care Excellence (2015) gestational diabetes mellitus diagnostic thresholds at this visit will be withdrawn from the study and immediately referred back to their antenatal team for follow-up. †Obtained from medical notes.

OGTT, oral glucose tolerance test.

intact proinsulin, 32-33 split proinsulin and des 31-32 split proinsulin) will be assayed.

\section{Setting and site selection}

Recruitment of pregnant women will be via a study poster placed at antenatal clinics within Abertawe Bro Morgannwg University Health Board, Wales. Study procedures will be carried out at a single site (Joint Clinical Research Facility, Swansea University).

\section{Informed consent}

Informed consent for each subject will be obtained prior to initiating any trial procedures. Potential participants eligible to take part in the study will receive an invitation letter from their hospital consultant or midwife, along with a participant information leaflet and be given an oral explanation about the study from a research professional (usually a research nurse). Written informed consent is given by the participant by signing and dating a consent form which will be countersigned and dated by either a study nurse or principal investigator to confirm that the participant has the opportunity to ask questions and fully understands the nature of the study. Thereafter, a copy of the consent form will be given to each of the participants. Research professionals can facilitate the consent process for the study if authorised to do so on the site delegation log following appropriate training including good clinical practice (GCP). The consent process makes clear that the participant can withdraw from the trial whenever they wish without giving a reason and without affecting their future care in any way. The reasons for withdrawal will be documented, if known, and site staff will be encouraged to trace participants lost to follow-up and document the reasons for their loss whenever possible.

Each participant will receive an identification number to ensure confidentiality and any samples will be identified by only using only the identification number. Data will be recorded in a case record form.

\section{Patient population}

Pregnant women at 16-18 weeks gestation with at least one of the following risk factors for GDM will be studied. Those recruited will meet the following inclusion/exclusion criteria:

\section{Inclusion criteria}

- $\mathrm{BMI}>30 \mathrm{~kg} / \mathrm{m}^{2}$.

- Previous macrosomic baby $(>4.5 \mathrm{~kg})$.

- Previous GDM.

- Family history of T2DM (first degree relative with diabetes).

\section{Exclusion criteria}

- Subjects unable or unwilling to sign informed consent.

- Known previous diabetes mellitus or on treatment with metformin.

- Known chronic infection like hepatitis or HIV or chronic kidney, liver or heart disease.

- Previous bariatric surgery. 
Women who will be diagnosed with gestational diabetes at the first visit (16-18 weeks) will be withdrawn from the study and referred back to routine antenatal care.

\section{Study visits}

Participants will be seen on three different occasions.

The first two visits will be at 16-18 weeks and 24-28 weeks gestation. Participants will be asked to attend having fasted for 10 hours. On arrival at the clinical unit, a fasting blood sample will be taken. Patients will then be given a drink containing $75 \mathrm{~g}$ glucose. At $30 \mathrm{~min}, 1$ and 2 hours following the drink further blood samples will be taken. The $30 \mathrm{~min}$ sample has been included to allow capture of the peak plasma insulin response and for robust estimation of insulin sensitivity.

The third visit will be 6-week post delivery where participants will be asked again to attend having fasted for 10 hours. On this occasion, a fasting blood sample only will be taken. Additional data including birth weight of the baby, Apgar score at birth and medications including insulin doses just prior to birth will be collected.

\section{Laboratory measurements}

Laboratory measurements for glucose, insulin, C peptide and proinsulin (total and intact) will be carried out in the Good Clinical and Laboratory Practice Accredited Diabetes Research Unit Cymru Laboratory based at Swansea University.

Glucose samples will be taken into fluoride oxalate tubes and will be measured using a glucose oxidase assay (YSI 2300 Stat Plus, Fleet, Hampshire, UK). Insulin, C peptide, total and intact proinsulin samples will be taken into EDTA tubes and measured using specific immunoassays using chemiluminescent labels (Invitron, Monmouth, UK).

\section{Safety evaluations and data monitoring}

The data monitoring committee (DMC) will monitor the overall conduct of the trial, safeguarding the interests of the trial participants and assessing the safety and efficacy of the intervention. Patients identified as glucose intolerant at 16 weeks will be considered to have had pre-existing glucose intolerance and will be referred back to their antenatal team and excluded from further participation in the study. All serious adverse events (AEs) that occur during the study will be recorded and reported in accordance with local requirements and will be reported to the DMC. All AEs will be recorded on a case report form and reviewed as part of central data monitoring.

\section{Statistical analysis plan}

To address the primary objective and the first secondary objective, glucose measurements following an OGTT at 24-28 weeks will be used as the reference standard to classify GDM status.

Optimal cut-off points for proinsulin will be established by the receiver operating characteristic plot which plotted the proportion of true positives against the proportion of false positives. 95\% CIs for the area under curve, sensitivity and specificity will be calculated to assess the diagnostic accuracy of total and intact proinsulin at 16-18 weeks gestation.

The same approach will be used to address the second secondary objective, but with the use of insulin during pregnancy as the reference standard.

Logistic regression will be used to analyse the relationship of proinsulin concentrations at 16-18 weeks gestation and other individual risk factors for the development of GDM.

All the analysis and data preparation will be done using statistical analyses which will be performed by a statistician using SPSS V.22 which is validated statistical software for clinical trial studies.

Values will be checked for normality, applying suitable transformations as necessary. All statistical hypothesis tests will be performed at a $5 \%$ significance level. All available data from withdrawn subjects will be included in the analysis up to the time of withdrawal where possible.

\section{Sample size}

Sample size was estimated with Buderer's formula ${ }^{15}$ which used prevalence, level of clinically acceptable precision and a hypothesised level of sensitivity and specificity as the parameters for estimating sample size.

There were no direct estimates of GDM prevalence in the high-risk population. Prevalence of GDM in the Western world was estimated to be $\sim 10 \%^{1}$ and the relative risk of developing GDM in women with one of the risk factors $\left(\mathrm{BMI}>30 \mathrm{~kg} / \mathrm{m}^{2}\right.$ ) was $2.74 .{ }^{16}$ There are also reports of women with a family history of diabetes having a $>6$-fold risk of developing gestational diabetes. ${ }^{17}$ Therefore, we have estimated the prevalence of GDM in our target population to be $\sim 30 \%$. We took the conservative approach of basing our sample size estimation on hypothesised sensitivity rather than specificity. Since the estimated prevalence was lower than 50\%, this approach would give a higher estimate of the number to be included in the study.

As a meaningful screening test, a sensitivity of $90 \%$ for the circulating concentration of total and intact proinsulin at 16-18 weeks gestation for GDM is expected as established by glucose measurements following an OGTT at 24-28 weeks. The minimal clinically acceptable precision for estimate of sensitivity is $6 \%$. We also expect loss to follow-up to be less than $10 \%$.

Adjusted for possible loss to follow-up, a sample size of 200 will be required to estimate with precision of \pm 0.06 for an expected sensitivity of 0.9 with the proinsulin test, at the significance level of 0.05 , if the prevalence of GDM is not lower than $30 \%$.

\section{Patient and public involvement}

In preparation for this study, the Public Reference Group of the Diabetes Research Unit Cymru was consulted and their opinions sought on the concept of the study and the study design. 


\section{ETHICS AND DISSEMINATION}

\section{Research governance}

The study conforms with the Research Governance Frameworks for England and Wales, and the principles of GCP outlined by the International Conference on Harmonisation (http://www.ich.org/). The participating National Health Service (NHS) Health Board has given NHS permission and will be responsible for auditing the study.

\section{Publication}

In accordance with good practice, we have registered the GDM study in a public registry at International Standard Randomised Control Trial Number (ISRCTN 16416602). This is a diagnostic accuracy study and findings will be reported according to the Standards for Reporting of Diagnostic Accuracy Studies (STARD) guideline. ${ }^{18}$ We shall present study findings at national and international conferences and publish as widely as possible in open access, peer-reviewed journals. Any published results will be made available to study participants from their study nurse on request (this is made clear in the patient information sheet) and any results will also be made available on the Diabetes Research Unit Cymru website (www.diabeteswales.org.uk) with a direct link to any publication and with a summary in lay language.

\section{DISCUSSION}

A recent systematic review and meta-analysis ${ }^{19}$ have shown that there is no good evidence for any of the diagnostic criteria for early onset GDM and have suggested the use of a fasting glucose of $6.1-6.9 \mathrm{mmol} / \mathrm{L}$ in the first trimester. Others ${ }^{20}$ have tried to develop a prediction model for obese women at high risk of GDM to facilitate targeted interventions.

Current practice is that pregnant women with at least one risk factor for GDM have an OGTT at 24-28 weeks and a fasting glucose measurement 6 weeks post delivery. It is not unusual for some patients having an OGTT at 28 weeks to have glucose levels above $10 \mathrm{mmol} / \mathrm{L}$ indicating that they have been hyperglycaemic for some time and therefore it would be useful if we could reasonably identify at an earlier stage those women who will develop GDM and also those that would require insulin to control hyperglycaemia. This would help manage scarce resources, as those that are unlikely to require insulin could be followed up by experienced allied healthcare professionals like specialist midwives while those that require insulin could be followed up by diabetologists and/or diabetes specialist nurses earlier in their pregnancy. It will also be useful to revisit the pathophysiology of GDM with respect to the onset of insulin resistance and corresponding insulin response at the various stages of pregnancy. Moreover, proinsulin assays have become more specific; thus, proinsulin can now be reliably measured by well-characterised immunoassays. This paper summarises the current approved protocol.
Acknowledgements The authors would like to thank Abertawe Bro Morgannwg University Health Board and Swansea University for independent review and clinical sponsorship of the trial. They would also like to thank the Public Reference Group of the Diabetes Research Unit Cymru for their comments on the concept and design of the study.

Contributors RP, SDL and GJD were involved in developing the concept of the study. All authors developed the study design and protocol. WYC has provided the statistical advice. All authors have read and approved the final manuscript.

Funding This work is supported by a Pathway to Portfolio Grant from Abertawe Bro Morgannwg University Health Board (ABMUHB) and was reviewed by the Joint ABMUHB and Swansea University Scientific Review Committee before the funding was approved. This is an investigator-led study.

Disclaimer ABMUHB will not be involved in interpreting, writing up or publication of the final results.

Competing interests None declared.

Patient consent Obtained.

Ethics approval The Wales Research Ethics Committee (Panel 6) (Ref. 17/ WA/0194).

Provenance and peer review Not commissioned; externally peer reviewed.

Open access This is an open access article distributed in accordance with the Creative Commons Attribution Non Commercial (CC BY-NC 4.0) license, which permits others to distribute, remix, adapt, build upon this work non-commercially, and license their derivative works on different terms, provided the original work is properly cited, appropriate credit is given, any changes made indicated, and the use is non-commercial. See: http:// creativecommons.org/licenses/by-nc/4.0/.

\section{REFERENCES}

1. Centers for Disease Control and Prevention National Diabetes Fact Sheet. General information and national estimates on diabetes in the United States, 2007. Atlanta, GA: US Department of Health and Human Services, Centers for Disease Control and Prevention, 2008.

2. NICE Guideline NG3. Diabetes in pregnancy: management of diabetes and its complications from preconception to the postnatal period. 2015 https://www.nice.org.uk/guidance/ng3/chapter/1Recommendations\#gestational-diabetes-2

3. Benaiges D, Flores-Le Roux JA, Marcelo I, et al. Is first-trimester $\mathrm{HbA1c}$ useful in the diagnosis of gestational diabetes? Diabetes Res Clin Pract 2017;133:85-91.

4. Bellamy L, Casas JP, Hingorani AD, et al. Type 2 diabetes mellitus after gestational diabetes: a systematic review and meta-analysis. Lancet 2009;373:1773-9.

5. Metzger BE, Cho NH, Roston SM, et al. Prepregnancy weight and antepartum insulin secretion predict glucose tolerance five years after gestational diabetes mellitus. Diabetes Care 1993;16:1598-605.

6. Retnakaran R, Qi Y, Sermer M, et al. Glucose intolerance in pregnancy and future risk of pre-diabetes or diabetes. Diabetes Care 2008;31:2026-31.

7. Linné Y, Barkeling B, Rössner S. Natural course of gestational diabetes mellitus: long term follow up of women in the SPAWN study. BJOG 2002;109:1227-31.

8. Amara FE, Meleis ME, Seif MA, et al. Study of pro-insulin level and its role in a cohort of women with gestational diabetes in Alexandria, Egypt. J Diabetol 2011;2:4.

9. Ward WK, LaCava EC, Paquette TL, et al. Disproportionate elevation of immunoreactive proinsulin in type 2 (non-insulin-dependent) diabetes mellitus and in experimental insulin resistance. Diabetologia 1987;30:698-702.

10. Swinn RA, Wareham NJ, Gregory R, et al. Excessive secretion of insulin precursors characterizes and predicts gestational diabetes. Diabetes 1995;44:911-5.

11. Dornhorst A, Davies M, Anyaoku V, et al. Abnormalities in fasting circulating proinsulin concentration in mild gestational diabetes. Clin Endocrinol 1991;34:211-3.

12. Phelps RL, Bergenstal R, Freinkel N, et al. Carbohydrate metabolism in pregnancy: XIII. Relationships between plasma insulin and proinsulin during late pregnancy in normal and diabetic subjects. $J$ Clin Endocrinol Metab 1975;41:1085-91.

13. Kautzky-Willer A, Thomaseth $\mathrm{K}$, Ludvik B, et al. Elevated islet amyloid pancreatic polypeptide and proinsulin in lean gestational diabetes. Diabetes 1997;46:607-14. 
14. Genova MP, Todorova-Ananieva K, Atanasova B, et al. Proinsulin in healthy pregnancy, pregnancy with gestational diabetes and after delivery. Acta Medica Bulgarica 2014;41:13-21.

15. Buderer NM. Statistical methodology: I. Incorporating the prevalence of disease into the sample size calculation for sensitivity and specificity. Acad Emerg Med 1996;3:895-900.

16. Pirjani R, Shirzad N, Qorbani M, et al. Gestational diabetes mellitus its association with obesity: a prospective cohort study. Eat Weight Disord 2017;22.

17. Lin $\mathrm{PC}$, Hung $\mathrm{CH}$, Chan TF, et al. The risk factors for gestational diabetes mellitus: a retrospective study. Midwifery 2016;42:16-20.
18. Cohen JF, Korevaar DA, Altman DG, et al. STARD 2015 guidelines for reporting diagnostic accuracy studies: explanation and elaboration. BMJ Open 2016;6:e012799.

19. Immanuel J, Simmons D. Screening and treatment for early-onset gestational diabetes mellitus: a systematic review and meta-analysis. Curr Diab Rep 2017;17:116.

20. White SL, Lawlor DA, Briley AL, et al. Early antenatal prediction of gestational diabetes in obese women: development of prediction tools for targeted intervention. PLoS One 2016;11:e0167846. 